\title{
New Technology Trends in the Design of Autonomous Ships
}

\author{
G. Koikas \\ Dept. of Industrial Design and \\ Production Engineering \\ University of West Attica, Athens, \\ Greece
}

\author{
M. Papoutsidakis \\ Dept. of Industrial Design and \\ Production Engineering \\ University of West Attica, Athens, \\ Greece
}

\author{
N. Nikitakos \\ Dpt. of Shipping Trade \& \\ Transport \\ University of Aegean, \\ Greece
}

\begin{abstract}
The continued development of the technologies applied to ship systems has pushed the shipbuilding industry to shipbuilding, with fully modernized automation systems that allow them to be handled and constantly monitored in real time from everywhere on the planet.

So the main purpose of this paper is to present the historical evolution of automation systems in shipping and to analyze both the existing technologies that take place in autonomous ships and the future trends. However, the potential risksconstraints underlying the use of this technology will be analyzed, as well as the important elements that should be given special importance so that the community can obtain a fully clarified picture of the particularly brilliant and promising technology of autonomous ships.
\end{abstract}

The results of this paper provide the basis for further analysis of future autonomous naval systems and contribute decisively both to the promotion of autonomous shipping and to the further improvement and development of independent maritime systems by assessing possible threats to avoid accidents.

\section{Keywords}

Autonomous ship, Risk Analysis, Autonomy levels, Reliability of autonomous systems, Shore Control Centrer, Remote Operator, Cybersecurity, Maritime test area, Autonomous Eco-shipping.

\section{INTRODUCTION}

This paper intends to examine thoroughly the applications of the technology, which are used for the design and implementation of autonomous ships. A technology that appears to be constantly developing and established at a constant rate, while gradually aiming at the transition to a new technological era for the shipping industry (figure 1) [1]

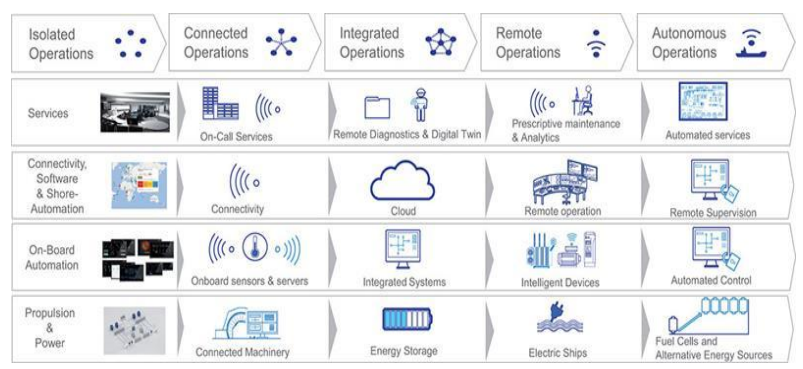

Fig 1: 5 steps of autonomous operations

In particular, the paper by identifying the evolution of automated navigation systems in shipping in the 21 st century, aims at highlighting the evolution of autonomous ships and the systems governing their technology, while emphasizing the analysis and evaluation of both the possible operational risks, as well as the estimated economic analysis of the operating costs of autonomous vessels. At the same time, it is investigating to what extent the current legislative framework makes the operation of such ships viable in the future.

Furthermore, the present and future developments of the technological progress of the autonomous systems are presented, together with guidelines for assuring the functionality and the reliability of the autonomous technology. Some pilot applications of autonomous ships are also presented, highlighting the interest of the scientific community in this new technology (figure 2). Finally, this paper examines the existence of a viable marine area of pilot testing of unmanned vessels in Greece, through the exploitation and evaluation of useful information and data drawn from approved carriers and specialized personnel, who work in this passage such as captains of ferry boats [2].

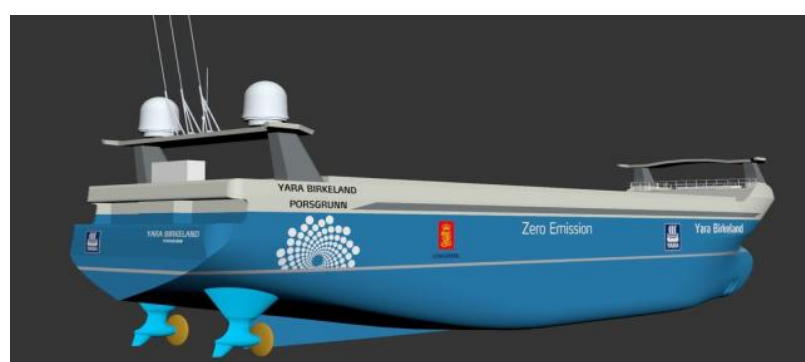

Fig 2: Autonomous ship project Yara Birkeland

This particular issue of paper was preferred to the highly scientific interest it presents, as it is essentially the latest in the innovation in the shipping industry. The prospects for the evolution of the technology of autonomous vessels are growing rapidly, as it was considered so far and judged by the current evolution of pilot testing control and navigation systems, that autonomous ships by 2030 will be adopted by a large number of shipping companies and will be a viable reality contributing to the transition to the new era of the shipping industry.

\section{Methodology}

The methodology that was developed in order to elaborate this paper was initially an introduction to the automatic navigation systems applied in the previous years, while presenting the existing evolved automated navigation systems, aiming to understand the developments and the changes that have occurred navigation technology in the shipping industry (figure 3) [3]. 


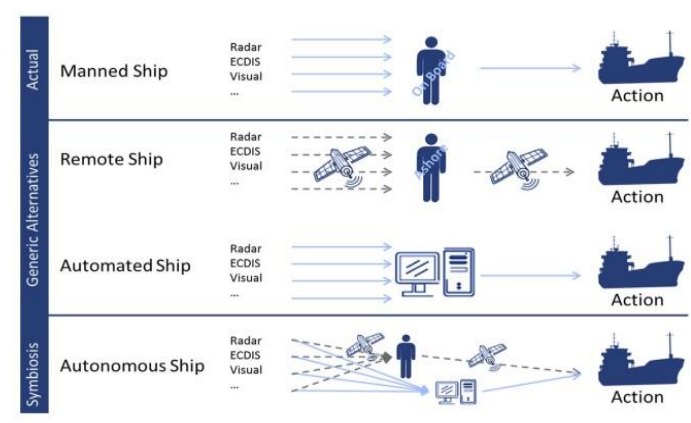

Fig 3: Progression from unmanned vessels to autonomous ships

Consequently an introduction to the autonomous ships is accomplished, as well as the autonomy levels that exist, listing the basic terms necessary for the understanding of the new autonomous technology, and the main differences between conventional, unmanned and autonomous ships (figure 4) [4].

\begin{tabular}{|c|c|c|c|}
\hline & Manned bridge & $\begin{array}{c}\text { Unmanned bridge - } \\
\text { crew on board }\end{array}$ & $\begin{array}{c}\text { Unmanned bridge- } \\
\text { no crew on board }\end{array}$ \\
\hline Decision support & $\begin{array}{c}\text { Direct control } \\
\text { No autonomy }\end{array}$ & Remote control & Remote control \\
\hline Automatic & Automatic bridge & Automatic ship & Automatic ship \\
\hline $\begin{aligned} \text { Constrained } \\
\text { autonomous }\end{aligned}$ & - & Constrained autonomous & Constrained autonomous \\
\hline $\begin{array}{r}\text { Fully } \\
\text { autonomous }\end{array}$ & - & - & $\begin{array}{c}\text { Fully } \\
\text { autonomous }\end{array}$ \\
\hline
\end{tabular}

Fig 4: Autonomy levels

In the following section, a thorough risk analysis and assessment is carried out on factors such as human errors, weather conditions, mechanical malfunctions and cybersecurity. However, the paper also extends to important areas such as assessing the viability of operating costs of autonomous vessels and assessing the existing legislative framework in order to ascertain, whether the operation and implementation of autonomous vessels is in line with applicable regulations or whether changes to the international conventions governing maritime law are required [5].

The next step analyzes the existing technology and the way an autonomous ship operates in conjunction with the presentation of the systems and the way they use intelligent data processing technology, such as artificial intelligence or Internet of Things (IoT) (figure 5) [6].

\section{IoT - Integrated Solutions \\ Public/Private Cloud Deployment Infrastructures}

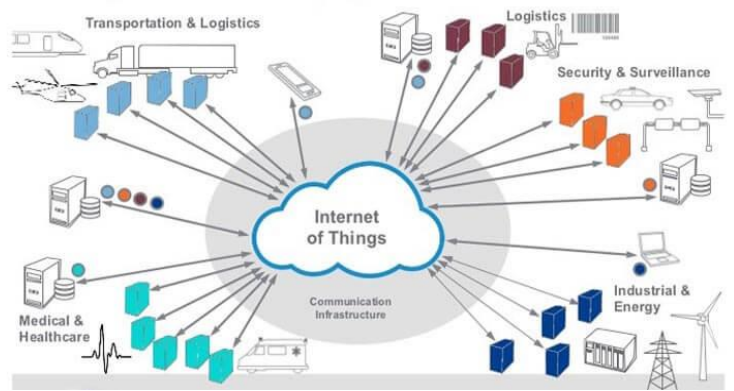

Fig 5: Internet of Thing Technology (IoT)
Additionally, specific guidelines are given for ensuring the functionality and reliability of the systems governing the operation of an autonomous ship, while detailing specific existing shipboard applications, as they have been made public by the responsible companies, that they have undertaken the management, design and implementation of such ships.

Also, this paper, it is investigated whether the creation of a maritime center for the testing of autonomous vessels at the specific sea passage in Greece, between the ports of Perama and Salamina is being investigated (figure 6) [7]. The evaluation takes place, by gathering important data and information, appropriate to shape the overall research outcome.

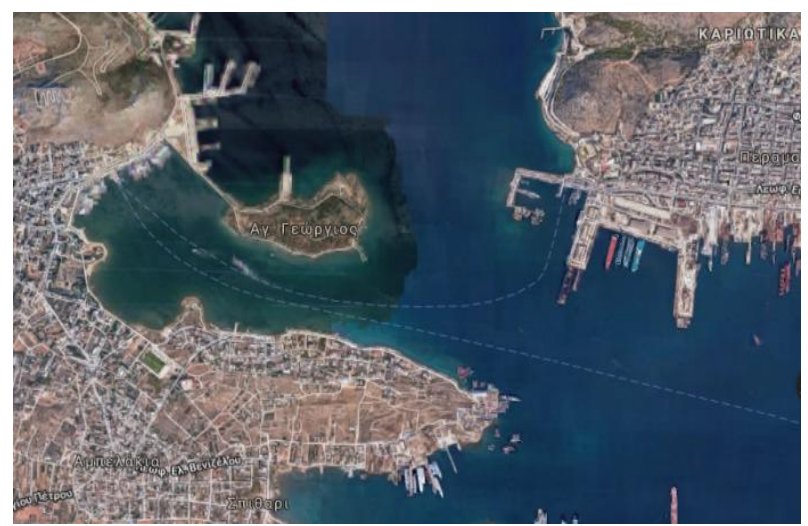

Fig 6: Satellite imagery of the Perama-Salamis passage

The final stage of the paper presents the future evolution of the technology of autonomous ships by presenting all the technologies and systems that were to be further developed and to assist in the optimal and efficient operation of the autonomous ships by 2030 (figure 7) [8].

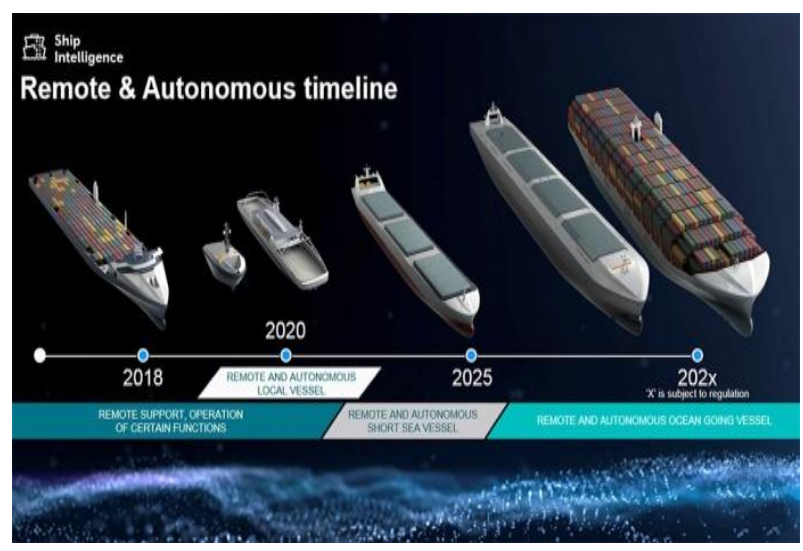

Fig 7: The future evolution of autonomous technology through the ages

The main sources of information and data mining, used to implement this paper, were the international program of the European Maritime Navigation through Intelligence in Networks (MUNIN) [9], as formulated by the French classification society BUREAU VERITAS, International Maritime Organization (IMO) [10], the Norwegian Forum for Autonomous Ships (NFAS) [11], as well as the technical manuals of both the construction and shipping companies Yara, Kongsberg, Rolls-Royce with project AAWA (Advanced Autonomous Waterborne Applications) [12], as well as the Norwegian classification society DNV-GL [13]. Lastly, the Greek meteorological website meteo, the database 
of the Hellenic Statistical Authority [14] and of course the testimonials of the people working in the studied Marine Test Area, also had an crucial contribution to the provision of information regarding meteorological, geographic and passenger data controls.

During the writing of the paper, however, some difficulties also emerged, such as the availability of information on the autonomous systems of unmanned ships, as the development of this new technology is at a relatively early stage and is constantly developing, while equally important observation is the fact that the results of the pilot tests of the applications of unmanned vessels have not been fully recorded, as they are expected to be completed by the end of 2019 .

However, the contribution of this paper to the wider scientific community was considered to be of paramount importance as it communicates, through the presentation of existing autonomous technology and the presentation of basic terms and systems that make up and govern unmanned ships and the tremendous development that has occurred in systems navigation at sea over the years, while also presenting the current development as well as at what stage the implementation efforts of testing unmanned ships.

In addition, it was particularly important the analyze and evaluation of the risks posed by the autonomous ships, as it provided substantial results on the suitability of such ships, the probability of undesirable situations, the impact that would arise and the safety levels that are capable to offer, to protect both human factor and the ship's own shipments themselves.

However, the paper has also been extended in terms of operating cost assessments, in order to assess the feasibility of the cost of implementing such a ship. Furthermore, the current legal framework has been evaluated so as to investigate, whether the operation and design of autonomous vessels it complies with the requirements and international regulations.

Finally, an important personal contribution to this paper was the attempt to study the creation of a maritime test area for autonomous vessels between the ferries of Perama and Salamis. This area was appropriately assessed using valid data, which were compared against prevailing conditions, geomorphological and meteorological data prevailing in the Norwegian and Finnish marine testing areas. Nonetheless, after analyzing and studying the reported data, this marine area was found to be inappropriate for the implementation of a test site as the basic conditions necessary to ensure the smooth and safe operation of autonomous vessels were not met, such as the increased maritime traffic and the lack of maritime infrastructure.

With regard to future research directions, this paper could provide the basis for future research into a number of important areas that could be as follows:

Initially, whether autonomous systems were improved to eliminate human risks and risks for the ship itself. It would also be of great importance to thoroughly investigate both the economic impact under the actual operating conditions of an autonomous ship, as well as the analysis of the future legal framework and the extent to which international regulations allow and enhance the applications of this technology.

Equally interesting would be the study and research of the new innovative systems that will be created, but also those that will be further developed and applied freely to newly build autonomous vessels by 2030 .
In addition, the development of autonomous eco-shipping is so advanced, that the creation of systems for the exploitation of renewable energy sources in autonomous and unmanned ships requires constant research, as it is a crucial and particularly critical factor, where it will in the future consider as a percentage, the future of shipping and the planet in general.

Another interesting filed of research is, whether the autonomous technology in the future will be assimilated by the structures of society, but also by the wider maritime community and to what extent it will essentially be implemented by the shipping companies, which would lead to useful conclusions on the intentions and trends of companies to invest in innovative technologies and lay the groundwork for the new era of autonomous shipping.

To sum up, a detailed analysis of the impact of the future operation of autonomous unmanned ships, both on the fundamental issue of human work and on environmental issues, could also be an extremely important and useful research.

\section{CONCLUSIONS}

Shipping industry is now very close to a transient state, that of autonomous shipping. All the technologies and systems that govern autonomous technology, are constantly developing and evolving rapidly, suggesting that the transition to this new era for the shipping sector, will take place presently in the coming years.

Both technology and shipping companies, seem to adopt future autonomous ship technology, seeing that it is the technological and environmental state-of -the-art, but mainly because the wider shipping industry is heading in this direction. But autonomous technology certainly raise questions about both human factor and ship security issues and these considerations extend to outstanding social issues, as is undoubtedly the extent to which human work is affected in the era of autonomous shipping and above all, the ecological impact of such ships on the environment.

Taking into account the analysis of possible risks in important areas such as the safety of human life and assessing the safety and navigation systems of the autonomous ships, it is stated that an autonomous unmanned ship has a lower risk profile than a conventional ship, makes it a highly secure and reliable, theoretical level so far, means of transporting people and commodities. On an economic level, the assessment of operating cost data shows that the operation of autonomous vessels will result in a significant reduction in costs for shipping companies, with the prerequisite that companies will invest in alternative sustainable biofuel technologies as a critical cost-cutting factor for an autonomous ship, is the combination of both cost savings and crew.

With regard to the legal framework, it is concluded that the current legal framework is insufficient for the start-up of autonomous vessels and that it is necessary to implement changes in international shipping conventions and regulations in order to have a sustainable scope of autonomous shipping.

In addition, the assessment of the case study of Greece, as a sea area of autonomous testing, after analyzing and studying the reported data, pointed out that this marine area was found to be inappropriate for the implementation of a test site as the basic conditions necessary to ensure the smooth and safe operation of autonomous vessels were not met, such as the increased maritime traffic and the lack of maritime infrastructure. 
Lastly, in the light of the current technological adequacy of the systems and pilot applications of autonomous technology, there is a tremendous development in the field of autonomous shipping and that, in the future, autonomous vessels will be fully viable and efficient in their operation but also in the crucial field of safety. The results of this paper lead to the conclusion that shipping is essential and of the utmost importance for both the environmental and human factor, to acquire an ecological character and to enter into a new technological era of evolution, where it will lead the autonomous shipping the next years at its peak.

\section{ACKNOWLEDGMENTS}

All authors would like to thank the University of West Attica and specifically the Post Graduate Program of Studies (MSc) "New Technologies in Shipping and Transport", for the financial support provided to them to undertake this research project.

\section{REFERENCES}

[1] https://new.abb.com/marine/generations/technicalinsight/ integrated-operations

[2] https://www.yara.com/newsandmedia/news/archive/2017/enova-to-support-thebuilding-of-yara-birkeland/

[3] http://www.unmannedship.org/munin/about/theautonomus-ship/

[4] http://nfas.autonomous-ship.org/resources/autonomdefs.pdf
[5] Maritime Unmanned Navigation through Int (Maritime Unmanned Navigation through Intelligence in Networks (MUNIN),European Commission, 2015) elligence in Networks. (2016, June). MUNIN. Retrieved from http://www.unmanned-ship.org/munin/

[6] https://www.globalmaritime.com

[7] https://www.google.com/intl/el/earth/

[8] http://www.thebrofessional.net/the-future-ofautonomous-cargo-ships/18

[9] http://www.unmanned-ship.org/munin/

[10] http://www.imo.org/en/OurWork/Security/Guide_to_Mar itime_Security/Documents/MSC.1CIRC. $1526 \% 20(\mathrm{E})$.pdf

[11] NFAS (2017) "Definitions for Autonomous Merchant Ships: NFAS Norwegian Forum for Autonomous Ships." White paper

[12] Jokoinen, E. P. (2016, September 21). Remote and Autonomous Ships - The next step. Hämtat från Rolls Royce, AAWA Position Paper. Retrieved from http://www.rolls-royce.com/ /media/Files/R/Rolls-: http://www.rolls-royce.com

[13] https://www.dnvgl.com/article/unmanned-ships-on-thehorizon-94273

[14] https://www.statistics.gr/statistics/-/publication/SMA06/- 